\title{
Les politiques de lutte contre le travail à bas
} salaire

Ce que nous enseigne l'expérience américaine

Policies to address low wage work: some lessons from the United States

experience

\section{Paul Osterman}

Traducteur : Hélène Boisson

\section{(2) OpenEdition}

\section{Journals}

Édition électronique

URL : http://journals.openedition.org/travailemploi/6610

DOI : 10.4000/travailemploi.6610

ISSN : 1775-416X

Éditeur

DARES - Ministère du Travail

Édition imprimée

Date de publication : 1 avril 2015

Pagination : 9-19

ISSN : 0224-4365

Référence électronique

Paul Osterman, «Les politiques de lutte contre le travail à bas salaire », Travail et Emploi [En ligne],

142 | avril-juin 2015, mis en ligne le 01 avril 2017, consulté le 19 avril 2019. URL : http://

journals.openedition.org/travailemploi/6610 ; DOI : 10.4000/travailemploi.6610 


\title{
Les politiques de lutte contre le travail à bas salaire : ce que nous enseigne l'expérience américaine $\left.{ }^{*}\right)$
}

\author{
Paul Osterman $\left.{ }^{* *}\right)$
}

Sur le marché du travail américain, 20 à 30\% des travailleurs occupent des emplois à bas salaire (selon le seuil retenu par l'auteur), qui se situent le plus souvent dans le secteur des services, tout en concernant un grand nombre d'activités et de professions. Dans ce contexte, la lutte contre les emplois à bas salaire et de faible qualité constitue un enjeu important pour les politiques publiques. Aussi, plusieurs leviers d'intervention sont-ils mis en auvre aux États-Unis : outre la fixation de normes en matière de rémunération (salaire minimum - fixé au niveau des États - et autres standards), les syndicats et d'autres organisations ciblant les travailleurs (community organizations et worker centers) peuvent se mobiliser pour soutenir les salaires. En parallèle, des outils innovants ont été développés à l'échelon local, tels que la mise en place de "salaires décents» s'appliquant aux salariés des collectivités locales ou à leurs prestataires, et la fixation de critères de sélection afin que les entreprises bénéficiant d'aides au développement économique s'engagent à faire progresser leur politique en matière d'emploi.

L'augmentation et la persistance des inégalités occupent une place de plus en plus importante dans le discours politique et économique aux États-Unis. $\mathrm{Au}$ début de la seconde période de son mandat, le président Obama, en parlant des inégalités, les a décrites comme «une menace fondamentale envers le rêve américain, envers notre mode de vie et ce que nous représentons à travers le monde ${ }^{(1)}$ »). Or, toute action engagée contre les inégalités passe nécessairement par une amélioration de la qualité de l'emploi, dans la mesure où, pour la plupart des gens, le travail constitue la principale source de revenus. Au cours des dernières décennies, le gouvernement fédéral, mais aussi de nombreuses collectivités locales, associations et community organizations $^{(2)}$ ont expérimenté différentes stratégies pour faire progresser la qualité de l'emploi. Le but de cet article est de présenter de façon synthétique à la fois l'ampleur du problème de

(*) Titre original en anglais : «Policies to address low wage work: some lessons from the United States experience». Traduction réalisée par Hélène Boisson.

(**) MIT Sloan School; osterman@mit.edu

(1) Citation extraite de l'article de Zachary A. Goldfarb, «With Democrats split on inequality issues, Obama shifts talk away from income gap», Washington Post, 4 juillet 2014, disponible en ligne à l'adresse : http://www.washingtonpost.com/ politics/with-democrats-split-on-inequality-issues-obamashifts-talk-away-from-income-gap/2014/07/04/102f1f32-02be11e4-b8ff-89afd3fad6bd story.html; consulté le 21 août 2015. (2) Pour une première définition de ce que recouvrent les community organizations, voir le Merriam-Webster, disponible en ligne ici : http://www.merriam-webster.com/ dictionary/community\%20organization; page consultée le 21 août 2015. l'emploi à bas salaire aux États-Unis et la variété des méthodes mises en œuvre pour tenter d'y répondre. Une première partie dressera un constat de la prévalence et des caractéristiques du travail peu rémunéré dans l'économie américaine. La suivante identifiera un certain nombre de leviers susceptibles de réduire l'étendue du phénomène. Enfin, la troisième et dernière partie mentionnera quelques-uns des défis qu'ont à affronter les politiques conduites.

\section{Le niveau du travail faiblement rémunéré dans l'économie américaine}

\section{Qui sont les travailleurs à bas salaire?}

Définir ce qu'est un emploi à bas salaire constitue une démarche normative. Une première solution consiste à se fonder sur des critères relatifs, comme le fait l'Union européenne en fixant un seuil de pauvreté au niveau des deux tiers du salaire moyen. La logique qui sous-tend cette approche est celle de l'inclusion sociale : les personnes dont les revenus sont excessivement inférieurs à la norme statistique risquent de ne pas être des citoyens à part entière, du point de vue économique comme du point de vue civique. Une approche alternative consiste à définir un standard absolu. Aux États-Unis, le «seuil de pauvreté» (poverty line) est une première étape en ce sens. Cette mesure de la précarité économique est largement utilisée; cependant, elle n'est pas sans 
poser problème, puisque l'ensemble des commentateurs, quelle que soit leur orientation, s'accordent à reconnaître que cette limite est excessivement basse ${ }^{(3)}$. Par exemple, en 2013, le seuil fédéral de pauvreté pour une famille de trois personnes (un parent et deux enfants) se situait à 18769 \$, soit une somme largement inférieure aux estimations des besoins de base. Pour remédier à ce problème, nous prendrons ici comme limite le salaire horaire avec lequel un actif travaillant à temps plein sur l'année complète assurerait à cette famille de trois personnes un niveau de vie compris entre 125 et $150 \%$ du seuil de pauvreté fédéral. Pour l'année 2013, ce salaire horaire était respectivement compris entre 11,27 et $13,53 \$$.

Sur la base de ces valeurs, en 2013, les adultes âgés de 25 à 64 ans étaient 20,6\% à toucher un salaire horaire inférieur à $125 \%$ du seuil de pauvreté pour une famille de trois personnes sur la base d'une année complète à plein-temps, et 30,8\% à gagner moins de $150 \%$ du salaire équivalant au seuil de pauvreté(4). En bref, entre 20 et $30 \%$ des travailleurs américains adultes exerçaient alors un emploi manifestement inférieur à la norme salariale. Plus des deux tiers $(67,4 \%)$ travaillaient à pleintemps, soit une proportion à peine inférieure à celle observée chez les actifs adultes dont les revenus dépassent le seuil de pauvreté retenu $(84,5 \%)$.

La répartition démographique est largement conforme à ce que l'on pouvait attendre : les femmes sont plus susceptibles que les hommes de se trouver en dessous des standards $(35,8 \%$ contre $26,2 \%)$ et les Africains-Américains (41,5\%) et Hispaniques (49\%) affichent des taux très supérieurs à ceux des Blancs non hispaniques $(24,1 \%)$. Naturellement, les Blancs non hispaniques étant les plus nombreux dans la population, ceux-ci sont malgré tout majoritaires en proportion de la population des travailleurs à bas salaires $(52,3 \%)$.

On s'intéressera enfin à la question de la mobilité permettant de sortir du marché des emplois à bas salaire. Si ces emplois n'étaient occupés que de façon transitoire par les actifs adultes et débouchaient ensuite sur de meilleures opportunités, que ce soit par le biais de la formation ou plus simplement de l'expérience acquise, leur existence serait moins préoccupante. Or, force est de constater qu'il en va tout autrement. Une importante série de recherches montre au contraire que la plupart des adultes occupant un emploi inférieur aux standards

(3) Voir par exemple, Greenberg M. (2009), It's time for a better poverty measure, Center for American Progress, Washington D. C.; memo disponible en ligne à l'adresse: https://www. americanprogress.org/issues/poverty/report/2009/08/25/6582/itstime-for-a-better-poverty-measure/; consulté le 21 août 2015.

(4) Ces calculs se fondent sur le Outgoing rotation groups of the Current Population Survey. Pour une description détaillée de la méthode de traitement des données utilisée, voir Osterman, Schulman (2011). définis ci-dessus restent prisonniers de ce type d'emplois (Holzer, 2004; Bradbury, 2011 ; KopczuK et al., 2010).

\section{Où travaillent-ils ?}

Pour s'attaquer au défi du travail à bas salaire, il convient d'abord de s'interroger sur la distribution sectorielle des emplois concernés. Le tableau 1 illustre leur répartition entre secteurs public et privé.

L'incidence des emplois à bas salaire est plus faible dans le public que dans le privé. Il n'en demeure pas moins vrai qu'un peu plus de $10 \%$ de l'ensemble des emplois à bas salaire s'y situent. Qui plus est, cette proportion augmenterait encore si l'on prenait en compte les entreprises privées sous contrat avec des institutions publiques, qui n'apparaissent pas dans les totaux du secteur public représentés dans le tableau. Plus de $40 \%$ des salariés à bas salaire du secteur public travaillent dans l'éducation, $8 \%$ dans la santé, $5 \%$ dans les transports, $4 \%$ dans les services sociaux, le reste étant dispersé dans les autres secteurs. Mettre en place des stratégies de transformation des emplois privés ne va évidemment pas de soi; en revanche, il devrait en principe être plus aisé d'élever les standards des emplois publics jusqu'à un niveau de revenu décent. Par ailleurs, le président Obama a également pris des mesures en faveur des salariés des sous-traitants passant des contrats avec le gouvernement fédéral.

Tableau 1 : Répartition par secteur, 2013

\begin{tabular}{|l|c|c|}
\hline & $\begin{array}{c}\text { Part du } \\
\text { secteur en } \\
\text { matière } \\
\text { d'emplois à } \\
\text { bas salaire }\end{array}$ & $\begin{array}{c}\text { Part du } \\
\text { secteur en } \\
\text { matière } \\
\text { d'emploi } \\
\text { total }\end{array}$ \\
\hline Secteur public fédéral & $1,3 \%$ & $3,2 \%$ \\
\hline Secteur public États & $3,5 \%$ & $5,2 \%$ \\
\hline Secteur public local & $6,4 \%$ & $8,8 \%$ \\
\hline Secteur privé à but lucratif & $82,8 \%$ & $75,7 \%$ \\
\hline $\begin{array}{l}\text { Secteur privé à but non } \\
\text { lucratif }\end{array}$ & $5,8 \%$ & $7,0 \%$ \\
\hline
\end{tabular}

Note : $1,3 \%$ des emplois à bas salaire se trouvent dans le secteur public fédéral, qui représente par ailleurs 3,2\% de l'emploi total.

Source: Census Outgoing Rotation Group, analyse des données par l'auteur. Voir note 3.

Le tableau 2 présente les activités du secteur privé où se concentrent les emplois à bas salaire. Seules sont représentées les activités totalisant au moins $3 \%$ de ces emplois. 
Tableau 2 : Répartition et incidence par branche d'activité (secteur privé), 2013

\begin{tabular}{|l|c|c|}
\hline & $\begin{array}{c}\text { Part de la } \\
\text { branche } \\
\text { en matière } \\
\text { d'emplois à } \\
\text { bas salaire }\end{array}$ & $\begin{array}{c}\text { Proportion } \\
\text { des salariés } \\
\text { de la } \\
\text { branche qui } \\
\text { sont à bas } \\
\text { salaire }\end{array}$ \\
\hline Production industrielle & $11,5 \%$ & $26,2 \%$ \\
\hline Construction & $5,0 \%$ & $26,4 \%$ \\
\hline Commerce de détail & $17,7 \%$ & $49,1 \%$ \\
\hline Transport et logistique & $4,1 \%$ & $30,7 \%$ \\
\hline Administration & $6,9 \%$ & $52,7 \%$ \\
\hline Éducation & $7,0 \%$ & $22,2 \%$ \\
\hline Santé & $12,6 \%$ & $29,1 \%$ \\
\hline Cafés restaurants & $11,2 \%$ & $72,7 \%$ \\
\hline
\end{tabular}

Note : $11,5 \%$ des emplois à bas salaire se trouvent dans la branche de la production industrielle, où ces salariés représentent $26,2 \%$ de l'emploi.

Source : Census Outgoing Rotation Group, analyse des données par l'auteur. Voir note 3 .

Le tableau ci-dessus met en évidence la dispersion des emplois à faible rémunération dans toute une série d'activités, des ouvriers aux employés, de l'industrie aux services. On remarque également la grande disparité des situations selon les branches concernées. Ainsi, dans la production industrielle, où les rémunérations sont élevées, l'incidence des bas salaires est inférieure à la moyenne; cependant, eu égard à ses effectifs considérables, la branche représente une part importante de l'ensemble des bas salaires. Les cafés-restaurants regroupent à peu près le même nombre de travailleurs faiblement rémunérés, mais il s'agit principalement d'une branche à faible rémunération, où l'incidence des bas salaires est particulièrement forte $(72,7 \%)$. Dans cette activité, comme dans celle du commerce de détail, le grand nombre de petits employeurs pose également des problèmes particuliers quant aux politiques à mettre en œuvre.

Le constat selon lequel $30 \%$ des adultes occupent un emploi synonyme de pauvreté constitue un précieux indicateur quant à la performance du marché du travail américain. Mais avant de songer aux actions à mener pour lutter contre la pauvreté laborieuse, on peut se demander si la proportion actuelle des bas salaires représente un progrès ou une dégradation par rapport à la situation antérieure, ou encore une stagnation. Si l'on constatait, par exemple, que la tendance est à une réduction constante de l'emploi mal payé, une action plus offensive apparaîtrait peut-être moins urgente.

Le tableau 3 représente la proportion de salariés à bas salaire en 2013, 2007 et 2001. Les années 2007 et 2001 sont des points hauts du cycle économique, et l'année 2013 semble s'approcher du haut du cycle actuel.

Deux conclusions peuvent être tirées des données. La première est que la situation ne va pas en s'aggravant; la crainte de voir une part croissante des emplois devenir de «mauvais» emplois est donc sans fondement. Ceci posé, le résultat à retenir est surtout l'absence d'amélioration. En effet, d'année en année, près de $30 \%$ des emplois occupés par un adulte ne lui permettent pas de gagner un salaire décent. Rien ne garantit, d'après les données dont nous disposons, que le temps, l'évolution du marché du travail ou la croissance économique permettront à eux seuls de rendre la situation moins préoccupante.

Tableau 3 : Incidence par année

\begin{tabular}{|l|c|c|c|}
\hline & $\mathbf{2 0 0 1}$ & $\mathbf{2 0 0 7}$ & $\mathbf{2 0 1 3}$ \\
\hline $\begin{array}{l}\text { Proportion de salariés à bas } \\
\text { salaire (au-dessous de } \mathbf{1 5 0} \% \\
\text { du seuil de pauvreté) }\end{array}$ & $29,6 \%$ & $30,1 \%$ & $30,8 \%$ \\
\hline
\end{tabular}

Note: Comme nous l'avons noté plus haut, le seuil de pauvreté défini au niveau fédéral se fonde sur des données de pouvoir d'achat. Il fait l'objet d'une révision annuelle afin de tenir compte de l'inflation. La plupart des chercheurs, considérant ce seuil comme dépassé et excessivement bas, se réfèrent à $150 \%$ de ce montant.

Source : Census Outgoing Rotation Group, analyse des données par l'auteur. Voir note 3 .

\section{Quels leviers pour réduire l'emploi à bas salaire?}

\section{La formation : une efficacité relative}

Dans le discours communément tenu aux ÉtatsUnis, le problème des bas salaires renvoie en premier lieu à la formation: la solution résiderait dans l'élévation du niveau de compétences des travailleurs. Cette idée entre en résonance avec la culture de la responsabilité individuelle, et a peu d'incidence sur les acteurs en place du marché du travail. Cependant, la question de l'efficacité réelle de cette option ne doit pas être éludée : investir davantage dans l'éducation et la formation permet-il de réduire efficacement l'incidence des emplois sous-payés ? La question est complexe, et requiert une réponse nuancée. Deux niveaux sont en effet à distinguer. $\mathrm{Au}$ niveau des individus, l'investissement dans les études constitue un choix rationnel. Au niveau de la société, il existe aussi d'excellentes raisons de tirer vers le haut le niveau général de formation et de compétences, depuis les avantages sociaux de l'instruction jusqu'à ses retombées économiques. Néanmoins, à un horizon raisonnablement proche, une stratégie purement tournée vers l'éducation et la formation ne saurait suffire à métamorphoser la répartition actuelle de la qualité des emplois.

Le premier constat - chaque individu a intérêt à améliorer sa formation générale ou professionnelle - s'impose au vu des données concernant la répartition des revenus en fonction du niveau de qualification. En effet, bien qu'ils aient subi une stagnation, les revenus des diplômés de l'enseignement supérieur restent largement supérieurs à ceux du reste de la population active; en outre, 
les diplômés sont moins exposés au chômage. Cependant, il faut comprendre que si ce résultat est valable à l'échelle d'un individu, sa généralisation à l'ensemble des actifs ne saurait éliminer une fois pour toutes les emplois à bas salaire. Chacun a intérêt à se former pour progresser plus vite dans la file d'attente; mais la file, elle, ne disparaît pas pour autant.

Ceci dit, les arguments ne manquent pas, à l'échelle de la société, pour investir durablement dans l'éducation et la formation. De nombreux travaux mettent en évidence l'effet bénéfique de l'instruction sur la qualité de vie, bien au-delà de son intérêt purement économique. Le progrès constant de la technologie exige une main-d'œuvre toujours plus qualifiée : ce processus entamé depuis un siècle ne semble pas près de prendre fin. Toutefois, cette avancée technologique s'accomplit sur le long terme et de façon continue, et il est difficile de déceler une accélération du phénomène. Cette observation a son importance à l'heure où est volontiers annoncée, à tort, une transformation radicale du marché du travail, le progrès technologique menaçant même de mettre les «bons emplois » hors de portée de la plupart des Américains (Osterman, Weaver, 2014; CAPPelli, 2015).

Plus généralement, un cadre politique misant uniquement sur l'éducation et la formation professionnelle ne permettrait pas de réduire la proportion des emplois de faible qualité. Une simple observation de bon sens montre que la demande de soins de santé, de services à la personne, de restauration et de tant d'autres activités synonymes de bas salaires, continuera à exister, indépendamment de l'élévation du niveau de compétences de la population active. Même si l'élévation du niveau d'instruction est susceptible à long terme de modifier la répartition sectorielle de l'économie, cette évolution ne s'effectue ni à une échelle, ni à un rythme permettant de modifier la vie quotidienne des travailleurs.

La meilleure preuve des limites d'une stratégie misant seulement sur la formation est précisément l'absence de baisse constatée de l'emploi à bas salaire alors même que le niveau de qualification de la population active s'est accru de façon constante au cours des dernières décennies. Ainsi, la proportion des 25-29 ans ayant fréquenté même brièvement l'enseignement supérieur est passée de $45 \%$ en 1991 à $58 \%$ en 2000 , pour atteindre $63 \%$ en 2012, mais l'emploi mal rémunéré n'a pas reculé.

\section{Les types d'intervention en faveur d'une amélioration de la qualité de l'emploi}

Il existe toute une série d'outils permettant d'infléchir la politique adoptée par les entreprises en matière d'emploi. Nous en dresserons ici une typologie sommaire. On commencera par distinguer ce qui relève de la fixation de normes ou standards, d'une part, de l'assistance technique ou de la mise en place de dispositifs spécifiques, d'autre part. La première catégorie regroupe par exemple l'action syndicale, la législation sur le salaire minimum et le salaire décent, ou encore les contrats de développement local (community benefit agreements ou $C B A$ ). La seconde catégorie comprend les plans de formation propres à un secteur d'activité, l'intervention d'intermédiaires sur le marché du travail et les différents types de services d'aide au développement des entreprises.

Il convient également de distinguer les mesures visant à améliorer la qualité des emplois existants (transformer les mauvais emplois en bons emplois) de celles qui ont pour but de créer des emplois de qualité. La première série de mesures consistera par exemple en un effort d'augmentation des rémunérations, ou de création d'une échelle des salaires s'appliquant à des emplois déjà existants, notamment dans le commerce, la santé où l'hôtellerie-restauration. La deuxième série comprend les dispositifs de développement économique utilisant les politiques de l'emploi pour attirer les bons emplois ou aider les entreprises à accroître leur compétitivité et, en conséquence, à préserver les emplois de qualité qu'elles offrent déjà.

Le tableau 4 présente les différentes mesures en fonction des caractéristiques ci-dessus.

Tableau 4 : Typologie des interventions en faveur de la qualité de l'emploi

\begin{tabular}{|l|c|c|}
\hline & $\begin{array}{c}\text { Mise en place de } \\
\text { normes }\end{array}$ & $\begin{array}{c}\text { Mise en place de } \\
\text { dispositifs }\end{array}$ \\
\hline $\begin{array}{l}\text { Améliorer } \\
\text { les emplois } \\
\text { existants }\end{array}$ & $\begin{array}{c}\text { Salaire minimum } \\
\text { Salaire de } \\
\text { subsistance } \\
\text { Syndicalisation }\end{array}$ & $\begin{array}{c}\text { Échelles de carrière } \\
\text { Intermédiaires du } \\
\text { marché du travail } \\
\text { Dispositifs sectoriels }\end{array}$ \\
\hline $\begin{array}{l}\text { Créer des } \\
\text { emplois de } \\
\text { qualité }\end{array}$ & $\begin{array}{c}\text { Community Benefit } \\
\text { Agreements } \\
\text { Incitations fiscales } \\
\text { contrôlées }\end{array}$ & $\begin{array}{c}\text { Plans par secteur } \\
\text { Consortiums } \\
\text { ou partenariats } \\
\text { sous l'égide des } \\
\text { entreprises ou des } \\
\text { syndicats }\end{array}$ \\
\hline
\end{tabular}

Les paragraphes suivants proposent une analyse plus détaillée des contenus correspondant aux différentes politiques, en les illustrant par quelques exemples.

\section{La fixation de normes : salaire minimum et autres standards}

Le travail de fixation de normes (standard-setting policies) a pour but de définir un niveau "plancher» de qualité de l'emploi. Les deux points forts de cette approche tiennent, d'une part, à son aspect relativement simple et direct, d'autre part, à sa portée potentielle, bien supérieure à celle des actions incitatives.

Les politiques de fixation de standards peuvent être classées en deux catégories : celles qui sont mises en place par le gouvernement (comme les salaires minima) et celles qui émanent de l'initiative privée 
(par exemple celle des syndicats). Si l'on s'intéresse d'abord aux politiques publiques, le standard le plus connu, et de loin, est le salaire minimum (minimum wage). La valeur réelle du salaire minimum fédéral se situe aujourd'hui bien en dessous du sommet atteint en 1968. Face à la récession de 2007-2008, certains États ont choisi de réagir : une trentaine d'entre eux ont fixé des minima supérieurs à ce que prévoit la loi fédérale. Le président Obama a proposé de revaloriser le minimum actuel, soit 7,25 $\$$ de l'heure, pour le porter à $10,10 \$$. En 2013, plusieurs États et municipalités ont considérablement augmenté les salaires minima locaux, l'exemple le plus marquant étant celui de la ville de Seattle, qui a choisi de le fixer à $15 \$$.

Or, la notion de salaire minimum ne correspond pas uniquement à un niveau de rémunération. En effet, une hausse du salaire minimum peut conduire les employeurs à modifier en profondeur leurs pratiques en termes de ressources humaines. Dans un autre contexte, celui de l'action syndicale, cette idée est déjà présente : c'est celle de l'«effet de choc» (shock effect) quand un syndicat est présent dans une entreprise. L'argument est que lorsque les salariés bénéficient de salaires garantis satisfaisants, les entreprises sont incitées à reconsidérer et à modifier leur système d'emploi et de production pour gagner en efficacité, la productivité qui en résulte permettant justement de garantir ces niveaux de salaire. Plus récemment, des théoriciens de l'économie, en étudiant la manière dont les entreprises décident du volume de formation offert à leurs employés, ont avancé que l'existence d'un salaire minimum, en resserrant la structure des salaires propre à l'entreprise, accroît du même coup les opportunités de formation offertes à ceux qui sont au bas de l'échelle (Acemoglu, Pischke, 1998).

Le gouvernement fédéral dispose d'autres outils que le salaire minimum pour imposer des normes en termes d'emploi. Le Fair Labor Standards Act (loi promulguée en 1938) exige le paiement des heures supplémentaires; la Wage and Hours Division du département fédéral du Travail se montre désormais plus résolue à faire appliquer ces dispositions. En outre, le président Obama a promulgué plusieurs décrets incitant les sous-traitants de l'État fédéral à améliorer la qualité de l'emploi : les contrats avec des entreprises contrevenant de façon répétée à la législation en la matière sont désormais rejetés. Plus spectaculaire, une décision récente du Conseil général du National Labor Relations Board rend la société McDonald's conjointement responsable des conditions d'emploi et de travail chez tous ses franchisés. Si cette décision se voit confirmée par le Labor Relations Board dans son ensemble (ce qui est probable) et par les tribunaux fédéraux (ce qui est moins sûr), elle aura un impact considérable sur les nombreux secteurs d'activité fondés sur le principe de la franchise, en permettant une meilleure application des normes et peut-être un certain nombre d'avancées syndicales.

\section{Le rôle des contre-pouvoirs : syndicats et autres acteurs sociaux}

Outre l'instauration d'un salaire minimum, une autre méthode a largement fait ses preuves pour améliorer les conditions d'emploi sur le marché du travail à bas salaire : l'action collective organisée pour contraindre les employeurs à faire progresser leur politique en matière d'emploi.

Ces dernières années, l'augmentation du taux de syndicalisation dans les secteurs à faible rémunération, et notamment ceux où les immigrés sont nombreux, constitue l'un des rares motifs de satisfaction pour les organisations syndicales. Le succès remporté par le mouvement Justice for Janitors en est un exemple remarquable; le syndicat de l'hôtellerie-restauration Hotel and Restaurant Workers Union (HERE) organise également des campagnes de ce type. Les syndicats porteurs de ces mouvements centrent explicitement leurs efforts sur les salariés mal payés, le plus souvent issus de l'immigration; dans le secteur des services, le Service Employees International Union (SEIU) a été l'un des rares syndicats américains à voir ses effectifs augmenter. D'autres organisations, comme l'American Federation of State, County and Municipal Employees (AFSCME), sont parvenues à fédérer les salariés les moins payés du secteur public et ont remporté un certain nombre de victoires.

Dans la lutte contre les bas salaires aux ÉtatsUnis, les principales limites de l'intervention syndicale résident dans son faible taux de réussite. Dans le secteur privé, le taux de syndicalisation a chuté de $25 \%$ en 1973 à $7 \%$ à peine aujourd'hui. S'il est difficile de disposer de données sur les retombées des campagnes de syndicalisation, des recherches récentes montrent que parmi les mouvements qui aboutissent à des élections syndicales (ce qui ne se produit qu'une fois sur deux environ), un sur cinq seulement débouche sur la signature d'un premier accord d'entreprise (FERGUSON, 2008).

Comme le réclamaient les syndicats, des formes alternatives d'organisation et de représentation des salariés peu rémunérés se sont récemment développées sur une base territoriale. On note tout particulièrement l'émergence de puissantes community organizations et de centres dédiés aux travailleurs (worker centers) (OSTERMAN, 2002; Fine, 2006).

Différents réseaux fédèrent à l'échelon national les community organizations qui interviennent localement auprès des travailleurs mal payés et mettent au point des actions autour de problématiques économiques. Particulièrement actives dans les luttes pour la revalorisation du salaire minimum fédéral et la promotion d'un salaire décent, elles s'efforcent aussi d'exercer un contrôle sur les subventions d'aide au développement économique versées aux entreprises. En outre, elles proposent 
des formations professionnelles et d'autres services destinés aux salariés peu rémunérés. Ainsi, l'un des plans de formation les plus efficaces du pays, le projet Quest ${ }^{(5)}$ a été mis en place par une organisation de la ville de San Antonio (Texas), puis le réseau regroupant les community organizations affiliées l'a ensuite importé à l'identique dans plusieurs autres grandes villes du sud-ouest des États-Unis.

Les worker centers sont des structures fournissant des services juridiques et sociaux aux travailleurs à bas salaire, souvent récemment immigrés, travaillant pour les petites entreprises qui emploient la majorité de cette main-d'œuvre bon marché et qui, dispersées, ne constituent pas des cibles intéressantes pour les syndicats traditionnels. Outre leur fonction de fournisseur de services, certains de ces centres s'efforcent de se substituer aux embauches directes en gérant des bureaux de placement, l'idée étant d'unifier les niveaux de salaire des emplois proposés. Ils montent par ailleurs des campagnes dénonçant les pratiques de tel ou tel employeur. Certains d'entre eux adoptent également un agenda législatif. Ainsi, le Workplace Project de Long Island est parvenu à imposer le New York Unpaid Wages Prohibition Act, une loi visant à empêcher les employeurs de diminuer des salaires promis aux populations vulnérables.

Il est clair qu'au niveau national, les worker centers ne sauraient obtenir des résultats spectaculaires. Cependant, si l'on fait la somme de toutes les formes de représentation passant par d'autres canaux que les syndicats classiques - community organizations et worker centers -, l'activité est importante. Une question centrale reste ouverte : dans quelle mesure ces nouveaux modes d'organisation des travailleurs peuvent-ils joindre leurs forces à celles des syndicats traditionnels, afin de peser davantage dans le rapport de forces et d'avoir un véritable impact?

Enfin, les campagnes ciblées sur une entreprise constituent un autre mode de pression. La plus connue est sans doute celle menée par un collectif associant des salariés des supermarchés Wal-Mart, des groupes de défense des intérêts locaux et des représentants syndicaux pour contraindre l'entreprise à améliorer les salaires et les avantages sociaux. Certes, le but ultime est d'aboutir à une organisation syndicale traditionnelle, ce qui n'est pas envisageable dans l'immédiat. À plus court terme, la campagne vise à mobiliser l'opinion publique, que ce soit par le biais d'articles de presse ou de journées de mobilisation des employés (one-day walkouts), afin de pousser Wal-Mart à modifier sa politique sociale (HocQuelet, 2014). Les campagnes de ce type se sont désormais étendues à toute une série de secteurs employant une main-d'œuvre bon marché,

(5) Quality Employment through Skills Training (voir présentation infra). notamment les restaurants (autour du Restaurant Opportunity Center) et les taxis. La dernière en date est celle lancée en 2012 par les employés des fastfoods, qui ont organisé cinq journées de débrayage et récemment conduit le conseil général du National Labor Relations Board à déclarer la société McDonald's coresponsable, avec ses franchisés, de la politique sociale que ceux-ci appliquent. Il est encore trop tôt pour savoir quelle sera l'efficacité des actions ciblant une entreprise en particulier. En règle générale, elles ne mobilisent qu'une très petite fraction des travailleurs concernés, et dépendent des syndicats officiels pour leur financement. Mais les entreprises, y compris Wal-Mart, font preuve d'une sensibilité accrue aux retombées possibles de telles actions en termes de relations publiques, et des avancées ont ainsi déjà été obtenues.

\section{Normes et innovation : les nouvelles stratégies locales}

Depuis quelques années, les dispositifs initiés aux Etats-Unis par les acteurs locaux (villes et États) témoignent d'une grande créativité. C'est à ces mêmes niveaux de décision qu'ont été expérimentées les formes les plus novatrices de fixation de normes salariales. Ces actions comprennent les campagnes en faveur du «salaire décent» et du relèvement du minimum fédéral, mais aussi les Community Benefit Agreements ( $f f$. infra), ou encore le conditionnement des aides au développement économique. Toutes contribuent dans l'ensemble du pays à un mouvement important et continu appelant à actionner des leviers politiques pour augmenter les salaires.

Les réglementations locales sur le salaire décent (living wage ordinances) garantissent un «minisalaire minimum». Elles fixent des standards de rémunération pour des groupes de salariés strictement définis, le plus souvent employés des collectivités locales ou prestataires de services travaillant pour ces collectivités. La première réglementation de ce type a vu le jour à Baltimore en 1994; aujourd'hui, plusieurs centaines, comparables, sont en vigueur à travers le pays. Elles varient considérablement dans leur structure et dans le statut des salariés couverts, la majorité concernant soit des contractuels employés par les municipalités, soit directement des agents municipaux, soit les deux. Un nombre plus restreint de ces règles salariales s'adressent à des entreprises recevant des aides publiques.

Les mouvements en faveur du salaire décent, comme les ordonnances qui en découlent, poursuivent des objectifs multiples. Au-delà de leur effet direct, ils visent notamment à encourager l'organisation des salariés et à imposer dans le débat public la question de l'équité économique. Si le résultat est évidemment difficile à mesurer, les campagnes engagées semblent jouer un rôle significatif. 
De plus en plus de travaux sont actuellement consacrés à l'évaluation des campagnes de promotion du salaire décent. On remarquera que même les plus sceptiques admettent l'efficacité des ordonnances locales sur le relèvement des salaires. Le débat porterait plutôt sur les éventuelles conséquences négatives qu'elles pourraient avoir sur l'emploi; mais là encore, les plus dubitatifs reconnaissent que même dans ce cas, les gains en termes de salaires compensent les coûts. On peut donc parler d'un bénéfice net du côté des populations faiblement rémunérées (pour une synthèse de cette littérature, voir THOMPson, CHAPMAN, 2006). Les études évaluant l'impact de ces mesures sur les entreprises ne décèlent pas d'effets négatifs importants; certaines suggèrent même que la hausse des salaires est un outil efficace pour réduire le turn-over. En résumé, les lois sur le salaire décent semblent remplir correctement leur mission, mais leur portée demeure limitée.

Suivant le concept du salaire décent garanti localement, on peut tenter de fixer des normes salariales et/ou de protection sociale à l'échelle d'un secteur d'activité entier. Ainsi, dans de nombreuses villes, des lois locales sur les salaires et les prestations sociales cherchent à encadrer les pratiques des «grandes surfaces» (big box retailers). Implicitement, ces dispositions visent souvent l'entreprise Wal-Mart. Elles suscitent toutefois d'importantes critiques, et il n'est pas rare de voir un conseil municipal les adopter avant que le maire lui-même oppose son veto. Ceci dit, elles participent néanmoins du vaste effort actuellement mené (et décrit plus loin) pour contraindre telle ou telle entreprise à améliorer la qualité de ses emplois.

Une autre initiative pour améliorer la qualité de l'emploi à l'échelon national comme local porte sur l'encadrement de l'attribution des aides au développement économique. Le soutien largement apporté aux employeurs par l'État comme par les communes se traduit par toute une série d'avantages fiscaux et de primes encourageant l'installation et la croissance des entreprises. Jusqu'à une époque récente, les termes de ces accords entre entreprises privées et institutions suscitaient peu d'intérêt et de débats dans l'espace public. Selon l'estimation la plus récente, 43 États, 41 municipalités et 5 comtés ont mis en place des conditions restrictives pour au moins un de leurs programmes de subventions (les villes ou les États pouvant mener de front plusieurs programmes de soutien aux entreprises, ceci ne permet toutefois pas d'estimer quel pourcentage de leurs programmes est soumis à des conditions salariales). En outre, dix États exigent des entreprises bénéficiant d'une subvention un rapport public indiquant le nombre d'emplois que ces fonds ont permis de sauvegarder ou de créer, ainsi que les salaires prévus pour chacun de ces emplois (LERoY, MATTERA, 2008).
On peut considérer les Community Benefit Agreements comme des dispositifs combinant l'effort de contrôle des subventions à l'installation et les campagnes en faveur du salaire décent. Le principe de ces accords est de repérer en amont un important projet de développement nécessitant l'accord des autorités municipales, puis de former un collectif d'organisations locales pour mener une négociation avec le porteur du projet sur un certain nombre de points : embauches locales, niveaux de salaires, mais aussi création de divers équipements destinés aux habitants : logements à des tarifs accessibles, facilités de stationnement, infrastructures de loisirs, etc. Dans le cas où un accord est signé, la coalition appuie ensuite l'initiateur du projet pour l'obtention des autorisations nécessaires. Parmi les localités les plus actives, citons la ville de Los Angeles, où des groupements organisés et soutenus par la $L o s$ Angeles Alliance for a New Economy (LAANE) ont négocié des accords au moment du développement de l'aéroport de Los Angeles, du Staples Center et du Century Boulevard. Des actions comparables ont été menées à Denver, Milwaukee, Boston, Seattle et Chicago. Aussi intéressantes que puissent être ces initiatives, elles ont aussi leurs limites. En général, elles ne profitent qu'aux riverains des grands projets locaux; d'autre part, leurs ambitions restent modestes en termes de niveaux de salaires. Elles constituent toutefois un outil original qui contribue au mouvement général associant pouvoir politique et organisations locales pour tirer vers le haut la qualité de l'emploi.

Enfin, un problème important se pose concernant la fixation de normes au bas de l'échelle salariale, celui de l'application effective des standards existants. En effet, certains défenseurs de ce mouvement font état d'un déclin des efforts de contrôle au cours des dernières années. Il est difficile d'étayer ce sentiment par des données, mais une récente synthèse des situations rencontrées à New York souligne bel et bien des problèmes réels, notamment quant au paiement des heures supplémentaires et au respect du salaire minimum( ${ }^{(6)}$. Remédier à cette situation relève de la responsabilité conjointe du gouvernement fédéral et de la direction du Travail de chaque État. Sous la présidence de Barack Obama, le département d'État chargé du Travail a renforcé son action de contrôle, tandis que certains États et municipalités, comme celle de Miami, ont récemment adopté des dispositions législatives pénalisant le non-respect du salaire minimum.

(6) Plusieurs enquêtes révèlent que $67 \%$ des salariés à domicile ne sont pas payés pour leurs heures supplémentaires; $59 \%$ des salariés des restaurants font face au même problème, tandis que les petits commerces de Brooklyn enfreignent de façon régulière la législation sur les salaires et la durée du travail. Voir Brennan Center for Justice (2006), Protecting New York's Workers, report, p. 2; disponible en ligne à l'adresse : http://brennan.3cdn.net/97857ff03d80aae74e_5lm6b9tdl.pdf; consulté le 21 août 2015. 


\section{Nouvelles politiques, nouveaux problèmes (7)}

Comme le montre clairement ce qui précède, une énergie considérable est actuellement consacrée, dans l'ensemble du pays, à améliorer la qualité des emplois à bas salaire. Cette activité intense, si elle se réfère à une ambition commune, se présente sous des formes variées. Pour rendre compte de ces différentes contributions et évaluer leur issue probable, il convient d'examiner en quoi consistent ces divergences. Deux axes seront ici distingués : on s'intéressera d'abord aux objectifs poursuivis, puis aux stratégies mises en place pour les atteindre.

\section{Des objectifs pluriels}

Pour mieux distinguer les différentes attentes des politiques conduites, on peut considérer conjointement deux des démarches collectives présentées plus haut: d'une part, les actions en faveur de la hausse du salaire minimum fédéral et, d'autre part, les campagnes sur le salaire décent initiées par des community organizations affiliées au réseau national de l'IAF (Industrial Areas Foundation). À première vue, ces deux initiatives ont de nombreux points communs dans la mesure où elles visent, l'une et l'autre, le relèvement des bas salaires par le biais d'actions législatives. Toutefois, les différences entre ces deux interventions sont importantes. En règle générale, les campagnes de promotion du salaire minimum sont menées par une coalition regroupant différentes structures: syndicats, community organizations, associations d'utilité publique ou organisations militantes. Leur objectif est simple : faire voter et appliquer une loi. L'alliance conclue pour l'occasion ne présente pas d'unité, et sa géométrie peut évoluer en cours de route, en fonction des problématiques abordées. $\mathrm{Au}$ contraire, l'IAF, tout en menant campagne pour l'établissement d'un salaire décent, cherche principalement à construire une capacité organisationnelle pérenne. Cette orientation est bien perceptible dans son slogan «le pouvoir d'abord, la politique ensuite» (power before policy). La lutte pour l'application de telle ou telle politique est d'abord considérée comme un moyen d'éduquer, de mobiliser les membres de l'organisation et d'étendre son champ d'action. Jusqu'à un certain point, ces différences importent peu; il s'agit bel et bien de faire progresser le niveau de rémunération des salariés.

C'est sur le long terme que les divergences apparaissent. En règle générale, une coalition de défense du salaire minimum n'a pas vocation à survivre à

(7) Cette section s'appuie sur un travail mené en collaboration avec Annette Bernhardt. la cause qui l'a fait naitre pour mener de nouvelles batailles. Ajoutons que la mise en place d'une telle plate-forme de revendications communes demande une bonne dose d'énergie, difficile à maintenir sur le long terme. Ce problème ne se pose pas de la même façon à l'IAF, conçue comme une organisation durable, appelée à de multiples interventions successives. Étant donné que le travail effectif au sein d'une coalition regroupant différents acteurs peut s'avérer difficile, l'IAF, qui se concentre fortement sur la mobilisation et l'organisation, a souvent tendance à faire cavalier seul, stratégie qui, à long terme, pourrait constituer un frein à son action.

En termes de stratégie, on peut effectuer une seconde distinction entre les campagnes explicitement concentrées sur la hausse des bas salaires, et celles pour qui la revalorisation du bas de l'échelle salariale n'est qu'un objectif parmi d'autres. C'est l'opposition classique entre action ciblée et action généraliste, qui peut être replacée dans le contexte plus large des politiques sociales, où l'on établit une différence entre les dispositifs restreints et ceux touchant un public plus large (SKостоL, 1991). Le régime national des retraites est un exemple type de dispositif qui participe à la redistribution aux EtatsUnis et est largement soutenu par la population. Parce que tout le monde peut en bénéficier, les politiques y sont largement favorables; le fait est qu'il a permis de réduire de façon drastique la pauvreté chez les personnes âgées. Pour cette même raison, le programme Medicare et, dans une moindre mesure, le système public d'éducation, jouissent d'un soutien comparable. La question est de savoir si on peut envisager des politiques du marché du travail pourvues des mêmes caractéristiques tout en étant capables d'atteindre leur objectif majeur, à savoir améliorer la qualité des emplois les moins bien rémunérés.

\section{Les acteurs engagés dans les initiatives d'amélioration de la qualité de l'emploi}

Entre ces deux types d'actions, on observe également des différences que l'on pourrait qualifier de tactiques plutôt que de stratégiques, encore qu'il ne soit pas toujours évident de distinguer ces deux aspects. Quoi qu'il en soit, les différences d'approche sont manifestes.

La base sous-jacente aux différentes actions peut varier; dans certains cas, elle est fortement liée à une identité ethnique, dans d'autres encore, à une identité locale, dans d'autres enfin, à un lieu ou à un cadre commun de travail. Le mouvement Justice for Janitors - une initiative particulièrement efficace basée à Los Angeles et destinée à améliorer les conditions de travail du personnel d'entretien - était au départ majoritairement constitué par des Latinos, et s'inspirait librement d'une culture acquise lors des mouvements de revendications des salariés 
agricoles. On se souvient notamment du slogan à succès «Si se puede» («Oui, on peut»). Or, cette dimension ethnique a contribué tout à la fois à entretenir le dynamisme et la solidarité au sein du mouvement, et à légitimer cette action aux yeux de l'opinion publique (MILKMAN, 2006). Au contraire, l'IAF est volontairement ouverte à tous sans distinction; elle évite de s'identifier à un groupe en particulier et cherche à mobiliser à l'échelle d'un quartier ou d'une paroisse (OSTERMAN, 2002). Les problèmes abordés par chacune des deux organisations sont généralement très ancrés dans la situation d'une aire géographique donnée. Pourtant, la lutte menée par les employés des fast-foods, les campagnes visant les supermarchés Wal-Mart ou encore les Restaurant Opportunity Centers sont autant de mouvements qui, en se concentrant sur un même cadre de travail, transcendent les distinctions ethniques et géographiques.

Une autre distinction porte sur la cible principale des actions : certaines visent avant tout à peser sur la décision publique, tandis que d'autres cherchent à susciter une réaction de la part des entreprises. Les actions prenant pour thème le salaire minimum, le salaire décent ou le développement équitable ciblent le gouvernement, tandis que la défense des salariés des fast-foods ou des magasins Wal-Mart vise les employeurs eux-mêmes. Naturellement, le but commun de toutes ces actions est de voir la qualité de l'emploi augmenter; les différences se situent du côté de la voie choisie pour y parvenir. Il faut encore noter que pour ainsi dire toutes les campagnes menées en direction des pouvoirs publics le sont soit à l'échelle des différents États, soit à l'échelle des municipalités. Ces initiatives locales sont-elles à même de se fédérer pour gagner une efficacité au niveau national, et de quelle manière cette convergence pourrait-elle s'organiser ? C'est ce que nous examinerons plus loin.

Enfin, ces différents types d'action peuvent être classés en fonction du lien entretenu avec les organisations syndicales. Comme nous l'avons rappelé, le militantisme syndical a été jusqu'ici le chemin le plus sûr pour faire progresser la qualité de l'emploi, aussi bien pour les employés des classes moyennes que pour ceux qui se situent au bas de l'échelle. Mais l'affaiblissement du syndicalisme traditionnel nous invite à prendre en compte d'autres formes de mobilisation et ce, y compris pour des campagnes que l'on peut considérer comme liées à des syndicats. Ainsi, une organisation syndicale du commerce et de la restauration comme la United Food and Commercial Workers Union a soutenu directement les campagnes visant la société Wal-Mart; dans l'hôtellerie, la Hotel and Restaurant Employees Union appuie les actions du Restaurant Opportunities Center. Bien qu'elle ne soit pas habilitée à mener des négociations, la Taxi Drivers Union est affiliée à la grande fédération syndicale
AFL-CIO ${ }^{(8)}$. Si les syndicats apportent leur soutien à de telles organisations, c'est, d'une part, au nom de la défense d'intérêts communs et, d'autre part, parce qu'ils voient en elles le moyen de gagner de nouveaux adhérents, ou d'accentuer la pression sur les entreprises dépourvues de représentants syndicaux, afin d'éviter qu'elles ne se livrent à un dumping salarial en versant des salaires inférieurs aux seuils syndicaux. Quant aux organisateurs des mouvements alternatifs, ils voient dans les syndicats une source non négligeable de ressources et de pouvoir. Néanmoins, certaines actions entretiennent des liens moins étroits avec les organisations syndicales. Les syndicats peuvent ainsi participer à telle ou telle coalition au niveau d'un État sans être pour autant la force motrice du projet. Dans certains cas, enfin, les community organizations se tiennent volontairement à distance des syndicats, que ce soit par souci d'indépendance ou en raison d'une mésentente ancienne entre milieu associatif et syndicats.

\section{Travailler avec les entreprises}

Tous les efforts décrits plus haut ont pour objet de faire pression sur les employeurs pour qu'ils améliorent la qualité des emplois proposés. Cependant, une solution alternative consiste à travailler main dans la main avec les entrepreneurs pour parvenir à ce même but. Ainsi, de nouveaux programmes, intitulés «intermédiaires» ou «sectoriels», se proposent d'améliorer la qualité de l'emploi en mettant en place, conjointement avec les entreprises, des perspectives d'évolution en interne pour les salariés les moins rémunérés. Ces efforts se présentent sous des formes diverses: catégories de salariés visés, institutions pilotant le projet, nature des services offerts peuvent varier. On retiendra cependant une volonté commune de privilégier les «bonnes pratiques», à savoir un ensemble d'éléments qui font l'intérêt de ces nouveaux programmes ambitieux et les différencient des plans de formation professionnelle classiques.

Les acteurs de ces nouveaux programmes ont compris que leurs efforts seront d'autant plus efficaces qu'ils auront su mettre en lien les deux parties prenantes du marché du travail: les employeurs d'un côté, les salariés de l'autre. Ils s'efforcent donc d'acquérir une réelle connaissance des besoins des entreprises en termes de ressources humaines, et même, dans certains cas, cherchent à accroître leur compétitivité. En bref, il ne s'agit plus de solliciter les entreprises sous l'angle de l'aide sociale ou des relations publiques, mais sous l'angle du business.

En outre, ces programmes centrés sur les bonnes pratiques s'engagent de façon importante pour leurs bénéficiaires, en rejetant les formations trop brèves et de qualité médiocre, les investissements à court

(8) American Federation of Labor - Congress of Industrial Organizations. 
terme, l'accompagnement sommaire à la recherche d'emploi. Au contraire, des formations longues, un engagement durable auprès des entreprises et des services d'assistance financière et de conseil véritablement adaptés sont mis en place.

Des différences non négligeables existent au sein de ces programmes. Nombreux sont ceux qui s'appuient sur les community colleges (établissements d'enseignement supérieur de premier cycle) pour le volet formation, et concentrent leurs efforts sur le soutien apporté aux stagiaires et sur la collaboration avec les employeurs. D'autres, moins nombreux, ont développé leur propre offre de formation. Les initiateurs et porteurs de ces projets varient également : associations locales, syndicats, community colleges, organisations patronales, ou encore gouvernement d'Etat. Enfin, certains de ces dispositifs s'adressent uniquement ou majoritairement aux salariés déjà en poste, tandis que d'autres sont accessibles aux demandeurs d'emploi.

Du côté des actions menées en collaboration avec les entreprises, deux grandes stratégies se dégagent. La première consiste à redéfinir les profils de poste afin d'établir une échelle des carrières, ou d'élargir à d'autres tâches les emplois existants. Ceci suppose que soit conduite une réorganisation du travail avec les instances managériales, ainsi qu'une formation et un accompagnement des salariés confrontés à ces nouvelles responsabilités, afin de faciliter leur progression au sein de l'entreprise. La seconde, moins ambitieuse, se borne à encourager les entreprises partenaires à rendre davantage de formations accessibles à leurs salariés les moins rémunérés, dans l'espoir que ceci suffira à faire avancer leur carrière.

Plusieurs évaluations nationales de ces programmes sont en cours; dans l'immédiat, il faut se contenter des quelques études prudentes consacrées à tel ou tel projet. Notons toutefois que les premiers retours semblent positifs. On prendra pour exemple l'évaluation par répartition aléatoire de trois programmes «sectoriels» offrant une formation de base adaptée au secteur concerné (en l'occurrence la santé, la production industrielle et les technologies de l'information) à des personnes présentant de multiples handicaps à l'embauche et gagnant en moyenne moins de $10000 \$$ par an avant d'entrer dans le dispositif (Maguire et al., 2010). Après avoir suivi l'ensemble du programme, les revenus du groupe de traitement dépassaient ceux du groupe témoin de plus de $4000 \$$.

Un autre exemple parlant est celui du projet Quest mené dans la ville de San Antonio, emblématique à maints égards des fameuses «bonnes pratiques». Un travail est mené auprès des employeurs pour anticiper leurs besoins en main-d'œuvre. Les entreprises sont souvent impliquées dans la mise au point de nouvelles offres de formation. Les bénéficiaires du programme sont correctement accompagnés par le biais de conseils personnalisés, mais aussi de petits coups de pouce financiers. Enfin, le programme se déploie sur une durée relativement longue: l'investissement sur les personnes concernées est donc significatif. Les participants au projet Quest gagnent en moyenne $5000 \$$ de plus que le groupe témoin (Lautsch, Osterman, 1998). Capital Idea, un programme similaire lancé dans la ville d'Austin (Texas), a abouti à des résultats comparables (KING et al., 2009).

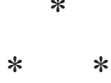

On peut se réjouir de ce qu'au cours des deux dernières décennies, bon nombre de stratégies de lutte contre les bas salaires ont déjà été expérimentées, ce qui a permis d'acquérir une solide connaissance de ce qui fonctionne et de ce qui pourrait fonctionner. L'autre sujet de satisfaction est le dynamisme avec lequel le problème est pris à bras-le-corps dans de nombreuses communautés. Outre sa créativité, l'ampleur géographique de ce mouvement est tout à fait remarquable : autant d'initiatives contrastent avec l'image d'un marché du travail américain purement libéral, et mettent en évidence l'importance des initiatives locales et de l'investissement des entreprises elles-mêmes dans les stratégies de lutte contre la mauvaise qualité de l'emploi.

En dépit de ces points positifs, une question épineuse demeure : quel est le résultat global de toutes ces actions ? Peut-on considérer qu'au bout du compte, les conditions de vie des travailleurs mal payés s'en trouvent transformées de façon significative ? Un certain nombre de signes suggèrent une réponse positive. À Seattle, les salariés à bas revenus disposent désormais d'un minimum horaire de $15 \$$, ce qui est bien au-dessus de la limite retenue dans cet article. Dans les localités où ont été signés des Community Benefit Agreements avantageux, des emplois de qualité sont créés qui ne l'auraient sans doute pas été précédemment. Mais le marché de l'emploi des États-Unis est immense, et le nombre d'adultes occupant un emploi souspayé, actuellement de l'ordre de trente millions, n'est apparemment pas en voie de diminution. Les louables efforts que nous avons décrits ne suffisent pas à inverser la tendance.

Pour espérer agir avec efficacité, tous les efforts locaux doivent se greffer sur une politique d'envergure nationale. L'expérience a montré que l'on peut y parvenir de deux manières : soit par un mouvement syndical fort, doté d'une réelle puissance d'action, soit par une politique fédérale résultant de la conviction des élus - ou plus vraisemblablement, des pressions exercées sur eux par différents groupes d'intérêts, comme ceux qui font valoir les droits 
des minorités raciales, des femmes, des personnes handicapées ou des homosexuels. Aujourd'hui, le syndicalisme n'est plus en position de force comme il a pu l'être par le passé; isolés, les défenseurs des travailleurs à bas salaires agissent en ordre dispersé.
Les organisateurs de ces initiatives parfois impressionnantes doivent chercher à unir les efforts de tous et à s'assurer un large soutien de la base. Ce n'est qu'en relevant ce défi que ces acteurs de terrain rendront possible une évolution en profondeur.

\section{Bibliographie}

Acemoglu D., Pischke J.-S. (1998), «Why do firms train? Theory and evidence», The Quarterly journal of economics, vol. 113, $\mathrm{n}^{\circ}$ 1, pp. 79-119.

Bradbury K. (2011), «Trends in U.S. family income mobility, 1969-2006», Working paper, $\mathrm{n}^{\circ}$ 11-10, Boston, Federal Reserve Bank of Boston.

CAPpelli P. H. (2015), «Skill gaps, skill shortages and skill mismatches: evidence and arguments for the United States», Industrial and labor relations review, vol. 68, $\mathrm{n}^{\circ}$ 2, pp. 251-290.

Clymer C., Conway M., Freely J., Maguire S., Schwartz D. (2010), Tuning into local labor markets: findings from the sectoral employment study, Philadelphie, NYC, Oakland, Public/Private Ventures.

Ferguson J.-P. (2008), «The eyes of the needles: a sequential model of union organizing drives, 1999$2004 »$, Industrial and labor relations review, vol. 62, $\mathrm{n}^{\circ} 1$, pp. 3-21.

FINE J. (2006), Worker centers: organizing communities at the edge of the dream, Ithaca, ILR Press/Cornell University Press.

Hocquelet M. (2014), «Grande distribution globale et contestations locales: les salariés de Walmart entre restructurations discrètes et nouvelles stratégies syndicales», Travail et emploi, $\mathrm{n}^{\circ}$ 137, pp. 85-103.

Holzer H. J. (2004), «Encouraging job advancement among low-wage workers: a new approach», Brookings Institution Policy Brief, $\mathrm{n}^{\circ} 30$.

King C. T., Carter Smith T., Schroeder D. G. (2009), Evaluating local workforce investments: results for short- and long-term training in Austin (TX), report, Ray Marshall Center for the Study of Human Resources, presented to APPAM's $31^{\text {st }}$ Annual Research Conference Washington, D. C., November 2009.
KopczuK W., SAez E., Song J. (2010), «Earnings inequality and mobility in the United States: evidence from social security data since 1937», Quarterly journal of economics, vol. 125, $\mathrm{n}^{\circ}$ 1, pp. 91-128.

Lautsch B., Osterman P. (1998), "Changing the constraints: a successful employment and training strategy », in Giloth R. P. (ed.), Jobs and economic development: strategies and practice, Thousand Oaks, Calif., Sage Publications, pp. 214-233.

LeRoy G., MatTera P. (2008), Uncle Sam 's rusty toolkit: how proven state and local reforms can make federal economic development programs better for taxpayers, workers and the environment, Washington, D. C., Good Jobs First.

MiLKMAn R. (2006), L.A. story: immigrant workers and the future of the U. S. labor movement, New York, N. Y., Russell Sage Foundation.

Osterman P. (2002), Gathering power: the future of progressive politics in America, Boston, Beacon Press.

Osterman P., Weaver A. (2014), « Skills and skill gaps in manufacturing», in Locke R. M., Wellhausen R. L. (eds.), Production in the innovation economy, Cambridge, MA, MIT Press, pp. 17-50.

Osterman P., Schulman B. (2011), Good jobs America: making work better for everyone, New York, Russell Sage Foundation.

SкосроL T. (1991), «Targeting within universalism: politically viable policies to combat poverty in the United States", in Jencks C., Peterson P. E. (eds.), The urban underclass, Washington D. C., Brookings Institution, pp. 411-436.

Thompson J., Chapman J. (2006), «The economic impact of local living wages ", Briefing paper, $\mathrm{n}^{\circ} 170$, Washington D. C., Economic Policy Institute. 